\title{
Endogenous regulators of protein phosphatase-1 during mouse oocyte development and meiosis
}

\author{
Xia Wang ${ }^{1,3,4}$, Jason E Swain ${ }^{2,4}$, Mathieu Bollen, Xiao-Tie Liu, Dana A Ohl ${ }^{3}$ \\ and Gary D Smith ${ }^{1,2,3,4}$ \\ Departments of ${ }^{1}$ Obstetrics and Gynecology, ${ }^{2}$ Molecular and Integrative Physiology, ${ }^{3}$ Urology and \\ ${ }^{4}$ Reproductive Sciences Program, University of Michigan, Ann Arbor, Michigan 48109-0617, USA
}

Correspondence should be addressed to G D Smith, 6428 Medical Sciences Building I, 1301 E. Catherine St, Ann Arbor, Michigan 48109-0617, USA; Email: smithgd@umich.edu

M Bollen is now at Afdeling Biochemie, Campus Gasthuisberg University of Leuven, Herestraat 49, B-3000 Leuven, Belgium

$X$-T Liu is now at Department of Pediatrics/Division of Neonatal-Perinatal Medicine, UT Southwestern Med CTT, 5323 Harry

Hine Blvd, Dallas, Texas 75235-7216, USA

\begin{abstract}
Reversible phosphorylation, involving protein kinases and phosphatases (PP), is important in regulating oocyte meiosis. Okadaic acid (OA) inhibition of PP1 and/or PP2A stimulates oocyte germinal vesicle breakdown (GVB). In oocytes, PP1 is localized in the cytoplasm and nucleus, yet endogenous regulation of oocyte PP1 has not been investigated. The objectives of the study were to identify intra-oocyte mechanisms regulating PP1 during acquisition of OA-sensitive meiotic competence and meiotic resumption. Immunohistochemical studies revealed that GVB-incompetent oocytes contained equivalent cytoplasmic and nuclear PP1. Upon development of OA-sensitive meiotic competence, PP1 displayed differential intracellular localization with significantly greater nuclear staining with distinct nucleolar rimming compared with cytoplasmic staining. Germinal vesicle-intact oocytes contained neither nuclear inhibitor of PP1, nor PP1 cytoplasmic inhibitor-1 transcripts or proteins. Reverse transcription-PCR with PP1 cytoplasmic inhibitor-2 (12) primers and oocyte RNA amplified a predicted 330-bp product with the identical sequence to mouse liver I2. Oocytes contained a heat-stable PP1 inhibitor with biochemical properties of 12. Phosphorylation of PP1 at Thr320 by cyclin dependent kinase-1 (CDK1) causes PP1 inactivation. Germinal vesicle-intact oocytes did not contain phospho-Thr320-PP1. Upon GVB, PP1 became phosphorylated at Thr320 and this phosphorylation did not occur if GVB was blocked with the CDK1 inhibitor, roscovitine (ROSC). Inhibition of oocyte GVB with ROSC was reversible and coincided with PP1 phosphorylation at Thr320. Increased oocyte staining of nuclear PP1 compared with cytoplasmic staining at a chronological stage when oocytes gain meiotic competence, and phosphorylation and inhibition of PP1 by CDK1 at or around GVB appear to be important mechanisms in regulating oocyte PP1 activity and meiosis. In addition, these studies provide further support for PP1 being the OA-sensitive PP important in the regulation of the acquisition of meiotic competence, nuclear events during meiotic arrest, and GVB.

Reproduction (2004) 128 493-502
\end{abstract}

\section{Introduction}

Pincus and Enzmann (1935) first described the process of spontaneous maturation of mammalian oocytes when they demonstrated that oocytes and/or oocyte-cumulus complexes could be removed from antral follicles and, under appropriate culture conditions, matured to metaphase II (MII). These oocytes are considered to display meiotic competence. However, oocytes from pre-antral follicles cultured under similar conditions do not mature, and thus exhibit meiotic incompetence. Acquisition of meiotic competence is correlated with oocyte size (Schultz \& Wassarman 1977), follicular morphology (Erickson \& Sorenson
1974) and species-dependent prepubertal chronological age (Schultz \& Wassarman 1977). Germinal vesicle breakdown (GVB)-competent and -incompetent oocytes display divergence in overall protein synthesis (Schultz \& Wassarman 1977) and expression of specific transcript and protein (Chesnel \& Eppig 1995, Harrouk \& Clarke 1995, Mitra \& Schultz 1996). Importantly, sensitivity to GVB stimulators, such as the protein phosphatase-1 (PP1) and the PP2A inhibitor, okadaic acid (OA) (Gavin et al. 1991, Hampl \& Eppig 1995), can differentiate between these oocyte subclasses. Currently, the mechanisms controlling acquisition of meiotic competence are poorly understood. 
Phosphorylation/dephosphorylation events are critical for resumption of meiosis (Bornslaeger et al. 1986). Specific phosphorylation events during meiosis are regulated, in part, by M-phase promoting factor (MPF) and/or mitogen activated protein kinase (MAP kinase) (Hashimoto \& Kishimoto 1988, Naito \& Toyoda 1991, Chesnel \& Eppig 1995). Serine/threonine protein phosphatases (PPs) have also been implicated in regulating meiosis (Rime \& Ozon 1990, Gavin et al. 1991, Schwartz \& Schultz 1991, Hampl \& Eppig 1995, Smith et al. 1998b). Okadaic acid stimulates GVB in starfish (Pondaven \& Cohen 1987), Xenopus (Goris et al. 1989), mouse (Alexandre et al. 1991, Gavin et al. 1991, Schwartz \& Schultz 1991), bovine (Levesque \& Sirard 1995) and macaque (Smith et al. 1998b) oocytes. These reports using OA, either through microinjection or in culture, suggest that PP1 and/or PP2A are involved in the G2/M cell-cycle transition. Recently, it has been demonstrated that both PP1 and PP2A are present within mouse (Smith et al. 1998a) and monkey (Smith et al. 1998b) oocytes. In meioticallycompetent germinal vesicle (GV)-intact oocytes PP1 is predominantly nuclear and appears to be the PP that regulates GVB, whereas PP2A is predominantly cytoplasmic (Smith et al. 1998a,b, Swain et al. 2003).

Protein phosphatase-1, but not PP2A, is sensitive to the heat- and acid-stable cytoplasmic inhibitors 1 and 2 (I1 and I2) (Cohen 1989). Inactivation of PP1 by I1 is dependent on 11 phosphorylation by protein kinase-A (PKA) (Nimmo \& Cohen 1978), whereas 12 does not require phosphorylation for PP1 inhibition (Nimmo \& Cohen 1978). Protein phosphatase- 1 is activated by a reaction controlled by glycogen synthase kinase-3 (GSK-3) phosphorylation of 12 (Hemmings et al. 1982, Resink et al. 1983). Because PP1 has been detected in the nuclei of numerous cell types (Jessus et al. 1989, Ohkura et al. 1989), experiments have focused on identification of a nuclear PP1 inhibitor. Nuclear inhibitor of PP1 (NIPP-1) has been isolated, characterized and cloned from thymus and liver (Beullens et al. 1992, Jagiello et al. 1995, Van Eynde et al. 1995). In addition, PP1 activity in somatic cells can be inhibited by phosphorylation at Thr320 (Van Eynde et al. 1995). This phosphorylation and inactivation of PP1 is regulated by cyclin dependent kinase-1 (CDK1) (Dohadwala et al. 1994), which oscillates during mitotic cell cycle progression (Kwon et al. 1997). M-phase promoting factor (Mitra \& Schultz 1996) and PP1 (Smith et al. $1998 a, b)$ translocate into oocyte nuclei in accordance with acquisition of meiotic competence, and remain predominantly nuclear until GVB. Because MPF is composed of CDK1 and cyclin-B, it is possible that oocyte nuclear MPF regulates nuclear PP1 phosphorylation and activity, and that this mechanism may be important in regulating phosphorylation of nuclear proteins in association with GVB. Such an event has yet to be reported.

The objectives of the present study were to elucidate intra-oocyte mechanisms of PP1 regulation in relation to cellular events influenced by the PP1/PP2A inhibitor, OA. Experiments were conducted to determine differential intracellular localization of PP1 and to investigate the role of endogenous cytoplasmic and nuclear inhibitors of PP1 and MPF-regulated PP1 phosphorylation and inactivation during acquisition of OA-sensitive meiotic competence and resumption of meiosis.

\section{Materials and Methods \\ Collection of mouse ovaries}

Ovaries were obtained from CF-1 female mice on day 11, 15 or 17 (day $0=$ day of birth) without hormonal stimulation or from adults primed with $10 \mathrm{IU}$ equine chorionic gonadotropin (eCG; Sigma Chemical Co., St Louis, MO, USA). Time points were selected in order to bracket a time when oocytes gain meiotic competence, as described by sensitivity to OA-induced GVB. For immunohistochemical studies, ovaries were fixed in $4 \%$ paraformaldehyde (prepared fresh daily), rinsed in PBS and embedded in paraffin.

\section{Immunohistochemistry (IHC)}

Fixed, paraffin-embedded ovaries were sectioned at 5-micron intervals, placed on superfrost-plus slides (Fisher Scientific, Itasca, IL, USA), deparaffinized, placed in $100 \mathrm{mM}$ glycine buffer ( $\mathrm{pH}$ 3.65) and microwaved for $10 \mathrm{~min}$ for antigen retrieval (Shi et al. 1995). To reduce background signal, samples were placed in Tris-buffered saline (TBS; $50 \mathrm{mM}$ Tris $\mathrm{pH}$ 7.6, $150 \mathrm{mM} \mathrm{NaCl}$ ) containing $5.0 \%$ dimethyl sulfoxide and $0.2 \%$ Tween-20 for 10 min, rinsed in TBS and incubated for $10 \mathrm{~min}$ in TBS containing $0.3 \% \mathrm{BSA}, 1 \mathrm{mg} / \mathrm{ml}$ sodium azide and $1.6 \%$ normal goat serum (Vector Laboratories, Burlingame, CA, USA). In addition, endogenous avidin and biotin were neutralized with an avidin/biotin blocking kit (Vector Laboratories). Slides were rinsed and incubated overnight with anti-PP1 $1_{\alpha}$ antibody diluted 1:250 at room temperature in a humidified chamber. Slides were then rinsed, washed (TBS $+0.05 \%$ Tween-20) and incubated with the biotinylated secondary antibody for $30 \mathrm{~min}$ at room temperature. Following secondary antibody exposure, slides were quenched in $3.0 \% \mathrm{H}_{2} \mathrm{O}_{2}$ in $90 \%$ methanol for $30 \mathrm{~min}$, then rinsed and incubated for $30 \mathrm{~min}$ in avidin-biotin conjugated to peroxidase (Vector Laboratories). After several rinses, sections were exposed to $0.025 \% 3,3^{\prime}$-diaminobenzidine (Dojindo Labs-Wako Chemical, Richmond, VA, USA), rinsed, counterstained with Mayer's hematoxylin (Sigma Chemical Co.), dehydrated by three changes of ethanol, then three changes of xylene, and mounted. Negative controls included (1) elimination of the primary antibody (not shown) and (2) non-immune rabbit serum in place of the primary antibody.

The ratios of $P P 1_{\alpha}$ nuclear-to-cytoplasmic staining intensity in GVB-incompetent and -competent oocytes 
were determined with a Leica DMR microscope $(\times 1000$; Leica Microsystems Inc., Bannockburn, IL, USA), using image acquisition with a SPOT-RT digital camera (Diagnostic Instruments Inc., Sterling Heights, MI, USA) and subjected to computerized densitometry (Scion Image, Scion Corp., Frederick, MD, USA). Briefly, oocyte micrographs had hematoxylin staining digitally removed prior to conversion to gray-scale (Adobe Photograph, Adobe System Inc., Mountain View, CA, USA). Regions of interest containing $80-90 \%$ of either cytoplasm or nucleus were used to calculate mean pixel intensity of $\mathrm{PP} 1_{\alpha}$ staining within the cytoplasm and nucleus. Differences in nuclearto-cytoplasmic staining intensity ratios of $\mathrm{PP} 1_{\alpha}$ in oocytes within ovaries isolated from 11-, 15- and 17-day-old mice were evaluated for statistical significance using Student's unpaired $t$-test. Differences were considered statistically significant at $P<0.05$.

\section{Oocyte collection and meiosis}

Fully grown GVB-competent oocytes were collected from eCG-treated (10IU) 19- to 23-day-old CF-1 mice utilizing a protocol approved by the University of Michigan Committee on the Use and Care of Animals. Oocytes were collected by puncturing antral follicles in HEPES-buffered human tubal fluid medium (H-HTF; Irvine Scientific, Santa Ana, CA, USA) with $0.3 \%$ polyvinylpyrrolidone (PVP; Sigma Chemical Co.). Oocytes used for Western blot analysis or RT-PCR were completely freed of attached cumulus cells via repeated mouth pipetting before freezing in liquid nitrogen and storage at $-80^{\circ} \mathrm{C}$. Cumulus-enclosed meiotic-competent oocytes were cultured in HTF $+0.3 \%$ BSA (control) or HTF containing $150 \mu \mathrm{M}$ of the CDK1 inhibitor, roscovitine (ROSC; Tocris, Ballwin, MO, USA) without oil overlay for $2 \mathrm{~h}$ at $37^{\circ} \mathrm{C}$ with $5 \% \mathrm{CO}_{2}$. This dose of ROSC was chosen based on dose-titration experiments and it completely inhibits oocyte GVB in our studies. A subset of ROSC-treated oocytes was removed from culture after $2 \mathrm{~h}$, washed extensively in $\mathrm{H}-\mathrm{HTF}+0.3 \%$ PVP and placed into HTF $+0.3 \%$ BSA for an additional $2 \mathrm{~h}$. At the end of culture cumulus cells were removed, GVB was assessed with an inverted microscope with Hoffman optics at $\times 400$, and oocytes were frozen in liquid nitrogen and stored at $-80^{\circ} \mathrm{C}$ until used for Western blot analysis.

\section{Oocyte RNA isolation and RT-PCR}

Eighty to one hundred oocytes were thawed in $20 \mathrm{IU}$ RNasin (Promega, Madison, WI, USA), pooled and lysed with 5 freeze/thaw cycles in liquid nitrogen. Total RNA was isolated as previously described (Heikinheimo et al. 1995, Smith et al. 1998a). Complementary DNA was synthesized using the Superscript Preamplification System for First Strand cDNA Synthesis reagents and methodology (Gibco BRL, Gaithersburg, MD, USA). Oligo-dT was used to prime reverse transcription reactions. For 11,12 , and NIPP-1 PCR, sense and antisense primers were designed from areas with least homology between related cDNAs (i.e. I1 and 12) to amplify size-discernible products with distinct restriction endonuclease digestion sites (Table 1). Each PCR was performed with five oocyte equivalents of CDNA, 20 pmoles of each primer, and a PCR cocktail (0.4 mM of each dNTP, $2.0 \mathrm{mM} \mathrm{MgCl} 2,10.0 \mathrm{mM}$ Tris- $\mathrm{HCl}$ (pH 8.3), $50.0 \mathrm{mM} \mathrm{KCl}$ and $2 \mathrm{IU}$ Taq DNA polymerase (Perkin-Elmer, Foster City, CA, USA)). In addition, control reactions were conducted consisting of (1) no template with primers and cocktail; (2) no primers with cDNA template and cocktail; (3) no template or primer with cocktail and (4) total RNA as template (equivalent to 5 oocytes) with primers and cocktail. Total RNA controls were only performed if amplification signals were previously identified in oocytes. Polymerase chain reactions entailed 1 cycle for $2 \mathrm{~min}$ at $94^{\circ} \mathrm{C}$; 35 cycles at $94^{\circ} \mathrm{C}$ for $1 \mathrm{~min}$, $55^{\circ} \mathrm{C}$ (NIPP-1), $57^{\circ} \mathrm{C}$ (I1) and $58^{\circ} \mathrm{C}$ (I2) for $1 \mathrm{~min}, 72^{\circ} \mathrm{C}$ for $1 \mathrm{~min}$ and $1 \mathrm{cycle}$ at $72^{\circ} \mathrm{C}$ for $2 \mathrm{~min}$.

Polymerase chain reaction products were separated on $2.0 \%$ low melting agarose gels (Sigma Chemical Co.) and isolated as previously described (Smith et al. 1998a). Portions of the amplified products were digested for $2 \mathrm{~h}$ with BamHI (11), HindIII (I2) or BamHI (NIPP-1). Undigested and digested PCR products, as well as PCR controls, were separated using $8 \%$ polyacrylamide gel electrophoresis and visualized with ethidium bromide staining. Reverse transcription-PCR experiments were performed in triplicate with each primer set to ensure repeatability of results. In addition, purified PCR products were sequenced by cycle sequencing (ABI Prism, Perkin Elmer) followed with product analysis using an Applied Biosystems Model 373A DNA Sequencer. Sequences obtained were

Table 1 Primers utilized for polymerase chain reactions for PP1 endogenous regulators in mouse oocytes.

\begin{tabular}{llcc}
\hline $\begin{array}{l}\text { PP1 endogenous } \\
\text { regulator }\end{array}$ & \multicolumn{1}{c}{ Primer* (Sense and Antisense) } & $\begin{array}{c}\text { Anticipated amplified } \\
\text { product (bp) }\end{array}$ & $\begin{array}{c}\text { Anticipated restriction } \\
\text { enzyme digest products (bp) }\end{array}$ \\
\hline NIPP-1 & 5' GCCTCAGCAAATTCCCATCG 3' & 402 & $331+71$ (BamHI) \\
Inhibitor-1 & $5^{\prime}$ TCCACCCGCTTCTTCTTGAC 3' & & $137+173$ (BamHI) \\
Inhibitor-2 & 5' CTCAAGTCCACACTGTCAATG 3' $^{\prime}$ & 310 & $154+176$ (HindIII) \\
& $5^{\prime}$ GTGGTAGCATGTGGGCTGAAA 3' & 330 & \\
\hline
\end{tabular}

*Sequence derivation: NIPP-1 (emb |Z50748.1| BTNIPPIMR); I1 (gb |J05592.1| RATPRIAA); I2 (gb |S79213.1| S79213) in GenBank. 
compared with existing sequences in GenBank with the use of BLAST via the INTERNET.

\section{PP1 cytoplasmic inhibitor assay}

In order to determine the presence of 11 and/or 12 at the protein level, human sperm, mouse brain and approximately 200 meiotic-incompetent and -competent oocytes were used to prepare heat-stable extracts as previously described (Smith et al. 1996). Pooled human sperm sonicates were prepared and used as the PP1 source in this assay (Smith et al. 1996, Vijayaraghavan et al. 1996). Boiled extracts (inhibitor source) and a PP1 source were incubated in duplicate for $10 \mathrm{~min}$ at $30^{\circ} \mathrm{C}$ before a standard PP assay was performed using ${ }^{32} \mathrm{P}$-phosphorylase-a as the PP substrate (Gibco BRL) (Smith et al. 1996). After incubation with ${ }^{32} \mathrm{P}$-phosphorylase-a, reactions were terminated with the addition of ice-cold $20 \%$ trichloroacetic acid (TCA) on ice for $10 \mathrm{~min}$; samples were centrifuged and supernatants were evaluated for ${ }^{32} \mathrm{P}$ release in response to the PP source with or without previous inhibitor source exposure. To identify the PP inhibitor (I1 or I2) present in mouse oocytes, heat stable sonicates were incubated in duplicate with $10 \mu \mathrm{m}$ ATP (Sigma Chemical Co.) with or without 1-2 mU PKA (Sigma Chemical Co.) for $10 \mathrm{~min}$ at $30^{\circ} \mathrm{C}$. Reactions were terminated in a $100^{\circ} \mathrm{C}$ water bath for $10 \mathrm{~min}$ and cooled at $30^{\circ} \mathrm{C}$. The protein phosphatase source was added before the PP assay was conducted. Positive control reactions for the assay of specific inhibitors utilized boiled extracts prepared from mouse brain (I1 and I2) (Endo et al. 1996, Kawai et al. 2001) and human sperm (I2) (Smith et al. 1996). Experiments were performed in duplicate.

\section{Electrophoresis and Western blot analysis}

Two hundred frozen cumulus cell-free fully grown GVintact and GVB oocytes were thawed in pre-warmed $2 \times$ SDS PAGE sample loading buffer $(80 \mathrm{mM}$ Tris $-\mathrm{HCl}$ $(\mathrm{pH}=6.8), 20 \%$ glycerol, $4 \%$ SDS, $4 \% \beta$-mercaptoethanol, $0.04 \%$ bromophenol blue), vortexed and placed on ice for $15 \mathrm{~min}$. Following sonication on ice, samples were denatured at $90{ }^{\circ} \mathrm{C}$ for $10 \mathrm{~min}$ and cooled on ice for $5 \mathrm{~min}$. Total protein from two hundred mouse oocytes was added per lane and separated by one-dimensional SDS-PAGE (Laemmli 1970). Approximately $5 \mu \mathrm{g}$ of rat and mouse liver protein were loaded per lane as positive controls for detecting NIPP-1. HeLa cell lysate was used as a positive control for recognizing PP1 and phospho-Thr320-PP1. Gels were equilibrated and transferred to Hybond-P PVDF transfer membrane (Amersham Life Sciences, UK) by Semi-Dry Electrophoretic Transfer (Bio-Rad Laboratories, Hercules, CA, USA) according to the manufacturer's instructions. After blocking, blots were incubated with either anti-NIPP-1 antibody (diluted 1:5000; kindly provided by Dr Bollen), or anti-phospho-Thr320-PP1 (diluted 1:1000; Cell Signaling Technology Inc., Beverly, MA, USA) antibody in TBST $(25 \mathrm{mM}$ Tris $\mathrm{pH} 7.6,125 \mathrm{mM}$
$\mathrm{NaCl}, 0.1 \%$ Tween-20) plus 5\% nonfat milk overnight at $4{ }^{\circ} \mathrm{C}$ with agitation. Blots were washed in TBST, incubated with anti-rabbit horseradish peroxidase-conjugated IgG (diluted 1:10000) at room temperature for $1 \mathrm{~h}$, washed again in TBST and developed with ECL Plus reagents (Amersham Life Sciences) according to the manufacturer's instructions. After phospho-Thr320-PP1 assessment, blots were stripped for $30 \mathrm{~min}$ at $50^{\circ} \mathrm{C}$ in a water bath with agitation using a stripping buffer $(62.5 \mathrm{mM}$ Tris- $\mathrm{HCl}(\mathrm{pH}$ 6.7), $100 \mathrm{mM} \beta$-mercaptoethanol and $2 \%$ SDS). Completely stripped blots were blocked in 5\% nonfat milk in TBST for $1 \mathrm{~h}$ at room temperature, then incubated with anti-PP1 $1_{\alpha}$ antibody (diluted 1:1000; kindly provided by Dr Angus Nairn, Rockefeller University) overnight at $4{ }^{\circ} \mathrm{C}$ with agitation, and processed further as described above.

\section{Results \\ PP1 localization and meiotic competence}

Increased PP1 in the oocyte nucleus may act as a regulatory mechanism during acquisition of meiotic competence. To further investigate this role of PP1, experiments were performed to determine nuclear-to-cytoplasmic PP1 staining intensity with respect to a chronological age when oocytes gain OA-sensitive meiotic competence (Gavin et al. 1991, Chesnel \& Eppig 1995). Ovaries from 11-dayold mice contained GV-incompetent oocytes with very little nuclear PP1 (Fig. 1A). On day 11, oocytes contained in secondary/preantral follicles had a nuclear-to-cytoplasmic staining intensity ratio of $1.1 \pm 0.1$ (arbitrary units, mean \pm S.E.; Fig. 1B). At 15 and 17 days of age oocytes began to display intense nuclear PP1 staining in accordance with approximately $10 \%$ and $60 \%$ acquisition of meiotic competence, respectively (Fig. 1A). On day 15, oocytes within follicles had a nuclear-to-cytoplasmic staining intensity ratio of $2.2 \pm 0.1$ (arbitrary units, mean \pm S.E.; Fig. 1B). On day 17, oocytes within follicles had a nuclear-to-cytoplasmic staining intensity ratio of $1.85 \pm 0.1$ (arbitrary units, mean \pm S.E.; Fig. 1B). Oocyte PP1 nuclear-to-cytoplasmic staining intensities on days 15 and 17 of age were significantly greater than on day 11 of age $(P<0.01)$.

\section{Cytoplasmic PP1 inhibitors and mouse oocytes}

Cytoplasmic PP1 activity can be regulated by inhibitor 11 and/or 12 (Ingebritsen et al. 1983). Because PP1 has a cytoplasmic, as well as a nuclear location, experiments were performed to elucidate the presence/absence of 11 and/or 12 at the transcript and protein levels. Reverse transcription-polymerase chain reaction with mouse brain mRNA as a template amplified the predicted $310 \mathrm{bp}$ product that contained a single BamHI digest site (Fig. 2A, lane $F$ and $G$ ). Using the same primers, with fully grown GV-intact mouse oocyte mRNA as a template, RT-PCR experiments revealed that oocytes do not contain the I1 transcript (Fig. 2A, lane E). A similar approach was 
A

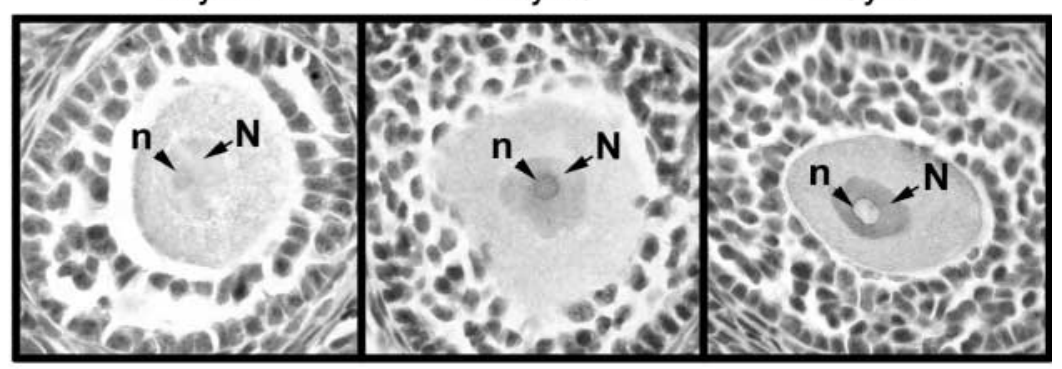

B

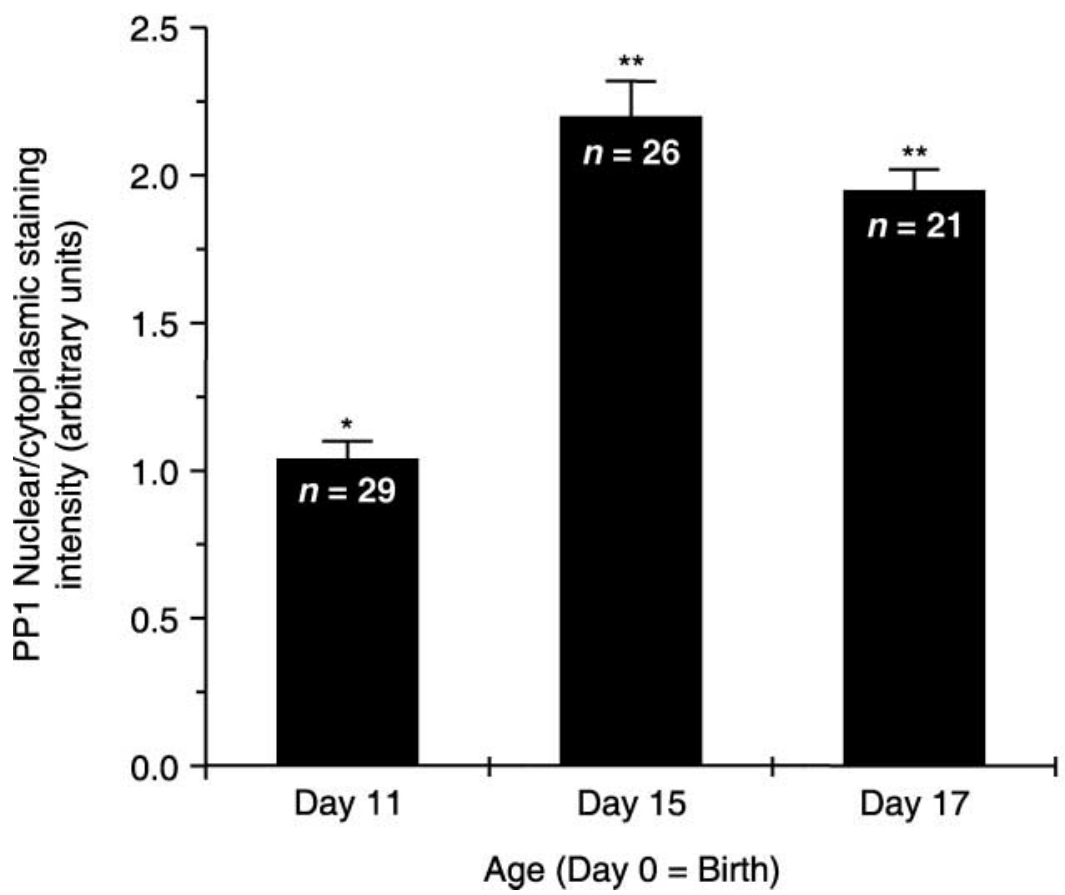

Figure 1 Intracellular trafficking of PP1 in mouse oocytes in relation to chronological age and acquisition of meiotic competence. (A) Representative immunohistochemical localization of PP1 in mouse oocytes during prepubertal ovarian development around the time of age-related acquisition of meiotic competence is shown. N, nucleus; $n$, nucleolus. Magnification $\times 1000$. (B) Protein phosphatase-1 nuclear-to-cytoplasmic staining intensity in mouse oocytes during prepubertal ovarian development around the time of agerelated acquisition of meiotic competence. At least 3 different ovarian sections were analyzed on each day. Twenty-nine (day 11), 26 (day 15) and 21 (day 17) individual oocytes were evaluated for nuclear and cytoplasmic staining of PP1 as described in Materials and Methods. Columns with different numbers of asterisks are significantly different $(P<0.01)$. employed to assess oocytes for the presence of 12 mRNA. The fully grown mouse oocyte cDNA produced by RTPCR with primers specific for $\mathrm{I} 2$ yielded a $330 \mathrm{bp}$ product (Fig. 2B, lane F) that contained an anticipated HindIII digestion site (Fig. 2B, lane G). Control PCR showed no reaction contamination. The sequenced mouse oocyte PCR product was identical to mouse liver 12 .

Due to the absence of specific 11 or 12 antibodies, oocyte PP1 inhibitors were evaluated at the protein level for the ability to inhibit PP1 activity. In this assay, we utilized the heat-stable characteristics and differential sensitivity to PKA to identify 11 and 12 activity, and to discriminate between these inhibitors. Inhibitor 1 and 12 are distinguishable by their response to phosphorylation. Inhibitory activity of $\mathrm{I} 1$ is stimulated after phosphorylation by PKA, whereas 12 is PKA-independent. Incubation of boiled human sperm extract with the PP source resulted in a PP activity that was $27 \%$ of the control activity (PP source/no inhibitor source; Fig. 3). Co-incubation with PKA did not influence the inhibitor potential of boiled human sperm extracts. This is in agreement with our previous identification and characterization of 12 in human, monkey and bovine sperm (Smith et al. 1996, Vijayaraghavan et al. 1996). Incubation of boiled mouse brain extract with the PP source resulted in a PP activity that was $85 \%$ of the control activity. When PKA was co-incubated with boiled mouse brain extract a fourfold increase in the inhibitory potential of this extract was observed. These controls, human sperm containing 12 and mouse brain containing $\mathrm{I} 1$ and $\mathrm{I} 2$, performed as expected in validation of the assay's ability to identify heat-stable PP1 inhibitors and to differentiate between 11 and 12 activity. Both meiotic-incompetent and -competent mouse oocytes contain PP1 cytoplasmic inhibitor activity (Fig. 3). In the absence of PKA, boiled meiotic-incompetent and -competent oocyte extracts resulted in PP activity that was $81 \%$ and $74 \%$ of control activity respectively. Co-incubation of either meiotic-incompetent or -competent extracts with PKA did not alter their inhibitory activity. Thus, the biological properties of the meiotic-incompetent 
A

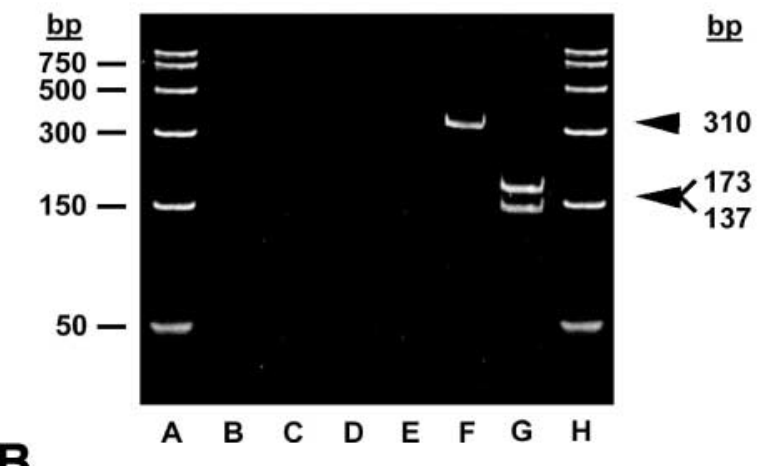

B

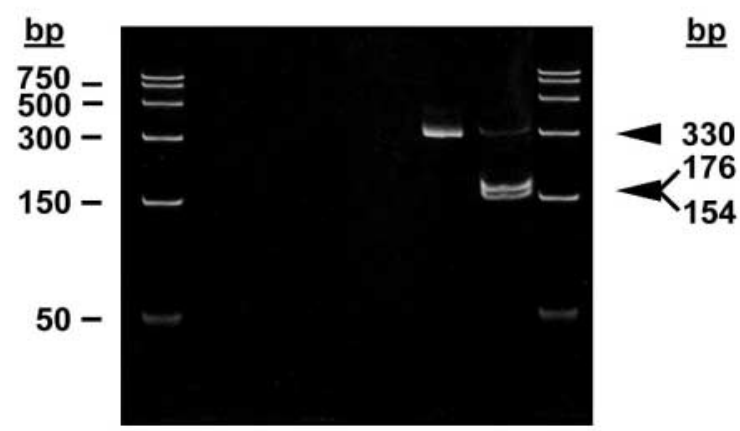

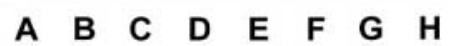

Figure 2 (A) Absence of the heat- and acid-stable PP1 inhibitor I1 mRNA in mouse oocytes: polyacrylamide gel electrophoresis of PCRamplified fully grown GV-intact oocyte and brain CDNAs using primers designed from rat brain I1 sequence. Lanes $A$ and $H, P C R$ markers; lane $\mathrm{B}$, primers, no cDNA; lane $\mathrm{C}, \mathrm{CDNA}$, no primers; lane $\mathrm{D}$, no cDNA, no primers; lane $\mathrm{E}$, mouse oocyte cDNA and primers; lane $\mathrm{F}$, mouse brain $\mathrm{CDNA}$ and primers; lane $\mathrm{G}$, mouse brain cDNA and primers followed by BamHI digest. (B) Identification of mouse oocyte I2 mRNA by RT-PCR/polyacrylamide gel electrophoresis and CDNA sequencing: PAGE of PCR-amplified products of fully grown GV-intact oocyte CDNA using primers designed from rat brain I2. Lanes $\mathrm{A}$ and $\mathrm{H}, \mathrm{PCR}$ markers; lane $\mathrm{B}$, primers, no cDNA; lane $\mathrm{C}$, cDNA, no primers; lane $D$, no $c D N A$, no primers; lane $E$, mouse oocyte total RNA and primers; lane $F$, mouse oocyte CDNA and primers; lane G, mouse oocyte cDNA and primers followed by HindIII digest.

and -competent mouse oocyte PP cytoplasmic inhibitor resemble those of $\mathrm{I} 2$.

\section{NIPP-1 and mouse oocytes}

When one considers the previously reported intense PP1 nuclear staining within fully grown GV-intact oocytes (Smith et al. 1998a,b), questions arise regarding control of nuclear PP1 and the possible involvement of NIPP-1. Nuclear inhibitor of PP1 within oocytes was investigated at the transcript and protein levels. Primers for RT-PCR were designed based on the rat liver cDNA sequence. Amplification for the predicted $402 \mathrm{bp}$ was detected using mouse liver cDNA as a positive control (Fig. 4A, lane F). This RT-PCR product also contained the anticipated single BamHI site, that when cut yielded 331 and 71 bp products
No inhibitor source $=$ control

Inhibitor source/no PKA = I2

Inhibitor source/PKA = I1

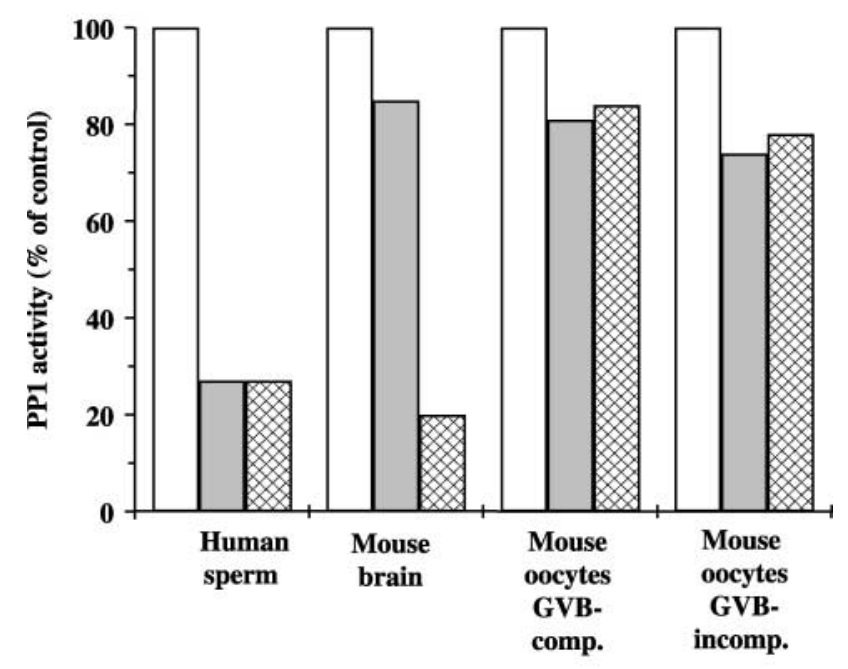

Inhibitor source

Figure 3 Representative identification and characterization of heatstable cytoplasmic PP1 inhibitor activity in mouse GVB-competent and -incompetent oocytes. Boiled mouse brain and human sperm extracts were used as positive controls for 11 and 12 activities respectively. Boiled mouse GVB-competent and -incompetent oocyte extracts were tested for I1 and 12 activity. Protein phosphatase activity was measured for a known PP1 source and considered as $100 \%$ activity (control). Boiled extracts were added to the known PP1 source in the presence and absence of PKA with resulting decreases in PP activity (\% of control) calculated for I1 and I2, respectively.

(Fig. 4A, lane G). As assessed by RT-PCR, mouse meioticcompetent oocytes did not contain mRNA for NIPP-1 (Fig. 4A, lane E). No amplification signals were detected in control reactions (Fig. 4A, lanes A-D). Western blots were performed with an antibody that previously had been described to recognize calf thymus NIPP-1a and NIPP-1b at 18 and $16 \mathrm{kDa}$, respectively (Beullens et al. 1992). In rat liver extracts, the major band recognized by the anti-NIPP-1 antibody corresponded to approximately $19 \mathrm{kDa}$. This immunogenically identified protein from rat liver matched the reported molecular mass of the purified 19 kDa PP1 inhibitory protein, which is believed to represent the rat liver homolog of calf thymus NIPP-1 (Zhao et al. 1996). In addition, this antibody also identified a similar protein band from mouse liver. However, protein extracts from fully grown GV-intact oocytes were devoid of NIPP-1 (Fig. 4B). Thus, fully grown mouse oocytes do not contain NIPP-1 transcript or protein.

\section{PP1 phosphorylation by MPF at GVB}

Inhibition of MPF's CDK1 subunit with ROSC blocks resumption of meiosis. Two hours after oocyte collection $88.4 \%$ of oocytes in control media $(n=301)$ underwent GVB. Conversely, $0.6 \%$ of oocytes treated with $150 \mu \mathrm{M}$ ROSC $(n=190)$ underwent GVB (Fig. 5A). This inhibitory 

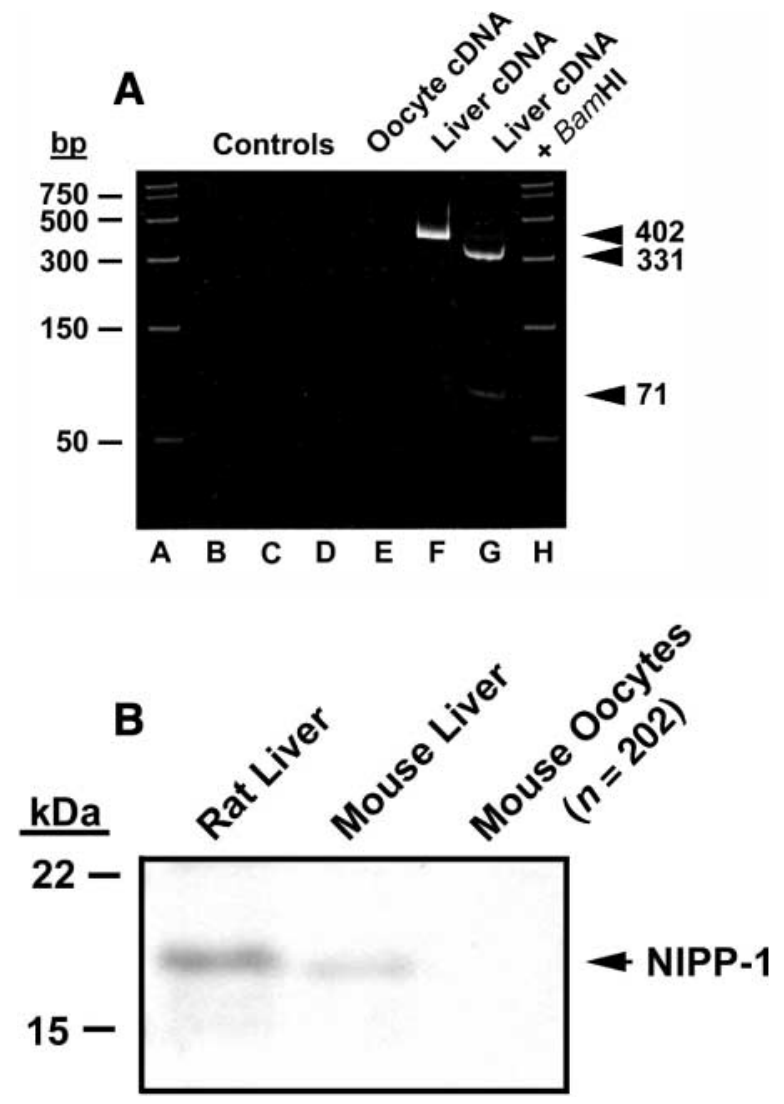

Figure 4 Identification of mouse oocyte NIPP-1 mRNA by RT-PCR/ polyacrylamide gel electrophoresis and immunoblot analysis. (A) Absence of NIPP-1 mRNA in mouse oocytes: polyacrylamide gel electrophoresis of PCR-amplified products of fully grown GV-intact oocyte and liver CDNAs using primers designed from the bovine thymus NIPP-1 sequence. Lanes A and H, PCR markers; lane B, primers, no cDNA; lane $\mathrm{C}$, cDNA, no primers; lane $\mathrm{D}$, no cDNA, no primers; lane $\mathrm{E}$, mouse oocyte $\mathrm{CDNA}$ and primers; lane $\mathrm{F}$, mouse liver $\mathrm{CDNA}$ and primers; lane $G$, mouse liver CDNA and primers followed by BamHI digest. (B) Immunoblot analysis of NIPP-1 in fully grown GV-intact mouse oocytes. Approximately $5 \mu \mathrm{g}$ of rat and mouse liver protein, as well as protein from 202 mouse GV-intact oocytes was loaded per lane. Antibody raised against bovine thymus NIPP-1 recognized a protein at $\sim 19 \mathrm{kDa}$ in positive control tissues, rat and mouse liver. Nuclear inhibitor of PP1 was not immunodetectable in mouse oocytes.

effect of ROSC was reversible since $86.7 \%(n=230)$ of ROSC-treated oocytes underwent GVB within $2 \mathrm{~h}$ following ROSC removal (Fig. 5A).

Protein phosphatase-1 and Thr320 phosphorylation of PP1 were assessed by Western blot analysis as represented in Fig. 5B. Protein phosphatase-1 was expressed at comparable levels in mouse oocytes at different developmental stages and under different treatments (Fig. 5B, top panel). Germinal vesicle-intact oocytes did not contain Thr320-phospho-PP1, while GVB oocytes did contain the phosphorylated protein. Blocking GVB with ROSC eliminated phosphorylation of PP1. If GV-intact oocytes were treated with ROSC for $2 \mathrm{~h}$, washed extensively, and cultured for an additional $2 \mathrm{~h}, 87 \%$ of oocytes underwent

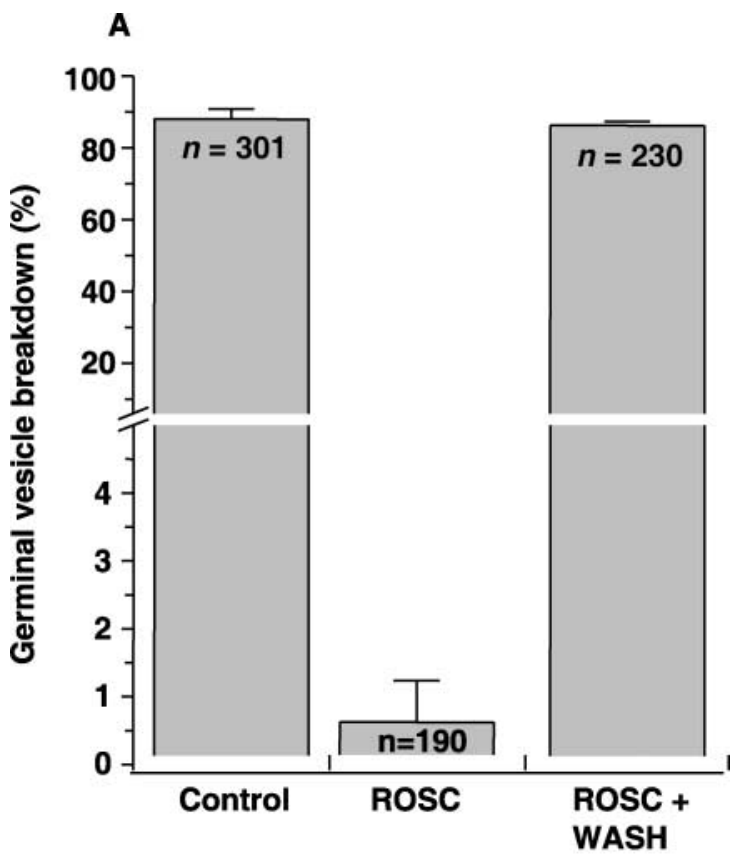

B
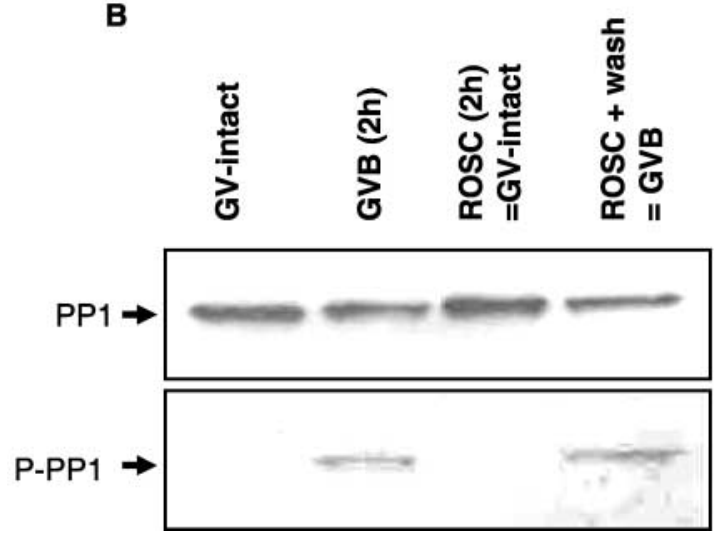

Figure 5 (A) Oocyte GVB in the presence of the meiotic inhibitor ROSC. Following $2 \mathrm{~h}$ of ROSC treatment oocytes were washed and placed into control media for an additional $2 \mathrm{~h}$. Data indicate that ROSC-induced inhibition of meiotic resumption is reversible. Experiments were performed in triplicate. (B) Representative immunoblot analysis of PP1 and phospho-PP1 (P-PP1) during mouse oocyte meiosis. Total protein extracts from 200 mouse oocytes were loaded per lane. CDK1 phosphorylated PP1 HeLa cell lysate was used as a positive control (not shown). The top panel displays PP1 expression. The bottom panel shows phospho-Thr320-PP1. Lane 1, GV-intact oocytes; lane 2, GVB oocytes; lane 3, GV-intact oocytes treated with $150 \mu \mathrm{M}$ ROSC for $2 \mathrm{~h}$; lane 4, GV-intact oocytes treated with $150 \mu \mathrm{M}$ ROSC for $2 \mathrm{~h}$ and transferred to fresh HTF media with $0.3 \%$ BSA for an additional $2 \mathrm{~h}$ to allow GVB. This experiment was performed in duplicate.

GVB, and GVB oocytes contained phospho-Thr320-PP1 (Fig. 5B, bottom panel).

\section{Discussion}

While the characterization, regulation and functional importance of numerous kinases are well defined in oocyte meiosis, knowledge of the specific role(s) of PPs 
and their regulation in oocytes is lacking. Results following treatment of oocytes with the PP1/PP2A inhibitors OA and calyculin-A (CL-A) suggest PP1 and/or PP2A are involved in GVB and progression to MII (Rime \& Ozon 1990, Gavin et al. 1991, Schwartz \& Schultz 1991, Hampl \& Eppig 1995, Smith et al. 1998b). Oocytes contain both PP1 and PP2A (Smith et al. 1998a,b). In this report we have begun to elucidate the PP1 endogenous regulatory mechanisms within mouse oocytes. Localization of PP1 in the nuclei compared with the cytoplasm is a potential mechanism of regulating intracellular PP1 activity and/or function. When oocytes begin to acquire OA-sensitive meiotic competence the differential intracellular localization of PP1 in nuclei is strikingly evident. Determination of this PP1 localization demonstrates a twofold increase in nuclear/cytoplasmic staining intensity at a chronological period when oocytes acquire OA-sensitive meiotic competence (Schultz \& Wassarman 1977, Gavin et al. 1991, Chesnel \& Eppig 1995). This differential localization of PP1 is independent of oocyte diameter and resident follicular stage. It is quite interesting that PP1 nuclear localization occurs at the same time as MPF is found to target to nuclei (Mitra \& Schultz 1996). One could hypothesize that this kinase/phosphatase pair may counteract phosphorylation of specific nuclear proteins involved with actual events of GVB. For example, hyperphosphorylation of nuclear lamins is an important step in initiating dissolution of the nuclear envelope (Peter et al. 1990). During oocyte development after meiotic-competence acquisition, the nuclear envelope may be maintained intact by nuclear PP1 counteracting the phosphorylation by MPF at the lamina level. This theory correlates well with findings of OA stimulation of GVB in the presence of unchanged MPF activity (de Vantery et al. 1996). Whether PP1 has the ability to regulate phosphorylation or oocyte nuclear lamins is currently under investigation.

The presence of both PP1 and PP2A within mammalian oocyte cytoplasm raises questions about the functional importance of this intra-oocyte location and the methods of PP1/PP2A activity regulation. Of particular importance are differential methods of regulating oocyte cytoplasmic PP1 or PP2A. One such pathway involves the heat- and acid-stable inhibitors, 11 and 12 . Currently no reports suggest the presence or absence of 11 in mammalian oocytes. Lack of I1 mRNA within fully grown oocytes, in conjunction with the inability to identify I1-like activity in germinal vesicle breakdown-incompetent and -competent oocytes indicate the absence of 11 in mouse oocytes. Presently, the only methods of assessing presence or absence of 11 protein are by chromatography purification and/or differential heat-stable PP1 inhibitor assays. Lack of an 11 specific antibody prevents immuno-detection. The heatstable PP1 inhibitor assay we have employed is commonly used to differentiate between I1 and I2 within cell and tissue extracts (Cohen 1978, Pondaven \& Cohen 1987, Vijayaraghavan et al. 1996, Smith et al. 1996). Absence of
I1 in mouse oocytes is in agreement with the report that starfish oocytes do not contain 11 (Pondaven \& Cohen 1987).

Starfish oocytes contain a heat-stable PP1 inhibitor with characteristics similar to I2 (Pondaven \& Cohen 1987). Here, we demonstrate that mouse oocytes also contain 12 as indicated by RT-PCR, cycle sequencing and inhibitor activity assay. As in starfish oocytes, the heat-stable inhibitor in mouse oocytes did not respond to preincubation with MgATP and PKA with respect to PP1 inhibitory activity, indicating that the heat-stable inhibitor is 12 . Characteristics of the mouse oocyte heat-stable PP1 inhibitor are the same for both meiotic-incompetent and -competent oocytes. A recent report indicates that 12 translocates from cytoplasm to nuclei of HeLa and PC3 cells (Leach et al. 2002). This raises the possibility that $I 2$ not only controls cytoplasmic PP1 activity, possibly in association with condensing chromatin, but also controls nuclear PP1 activity in the regulation of nuclear lamin phosphorylation and GVB. Additionally, because 12 requires no activation to inhibit PP1 these data would suggest that oocyte PP1 is susceptible to 12 inhibition and that levels of $\mathrm{I} 2$, or the state of 12 phosphorylation, may be important determinants of overall oocyte PP1 activity. It is useful to remember that when 12 is phosphorylated by GSK-3, PP1 activity is restored. We have recently demonstrated that mouse oocytes also contain GSK-3 (Wang et al. 2003). Whether GSK-3 and/or 12 activity change with respect to meiotic progression remains to be investigated. In addition, the possible role of this oocyte-derived PP1/I2 system in fertilization and early embryo development warrants future attention.

During mitosis, PP1 phosphorylation at Thr320 is associated with inhibition of PP1 activity (Dohadwala et al. 1994). In somatic cells PP1 becomes phosphorylated by CDK1 at the M2/G transition (Dohadwala et al. 1994, Kwon et al. 1997). Because inhibition of PP1 with exogenous means (such as OA administration) stimulates GVB, we surmised that an endogenous mechanism of inhibiting PP1 may be linked to GVB. At or around the time of GVB, PP1 becomes phosphorylated at Thr320. This PP1 phosphorylation is regulated by the CDK1 activity of MPF. Whether CDK1 directly phosphorylates the Thr320 residue and what proportion of oocyte PP1 becomes phosphorylated is not known at this time. This PP1 phosphorylation is not a time-dependent event in relation to removal of the oocyte from the follicle, but is dependent on MPF activity. These data also open up a new avenue of investigation involving MPF, PP1 and other nuclear proteins in the regulation of nuclear envelope integrity, breakdown, and reformation during oocyte meiosis.

In conclusion, differential intra-oocyte localization of PP1 to the nucleus when oocytes begin to gain meiotic competence suggests its importance in the regulation of meiotic competence acquisition, nuclear events during meiotic arrest, and GVB. In conjunction with GVB, PP1 becomes phosphorylated at Thr320, which is known to 
inhibit PP1 activity. This phosphorylation of oocyte PP1 is regulated by CDK1 activity of MPF. These studies have provided a link between MPF and PP1 at a developmental time-point when both these enzymes appear to be important in regulating oocyte GVB.

\section{Acknowledgements}

The authors would like to thank Dr Carrie Cosola-Smith for critically reviewing the manuscript and Dr Angus Nairn (Laboratory for Cellular and Molecular Neuroscience, Rockefeller University, New York) for kindly providing the PP1 antibody. This study was supported by $\mathrm{NIH}$ grant HD35125 (to G D S).

\section{References}

Alexandre H, Van Cauwenberge A, Tsukitani Y \& Mulnard J 1991 Pleiotropic effect of okadaic acid on maturing mouse oocytes. Development 112 971-980.

Beullens M, Van Eynde A, Stalmans W \& Bollen M 1992 The isolation of novel inhibitory polypeptides of protein phosphatase 1 from bovine thymus nuclei. Journal of Biological Chemistry 267 16538-16544.

Bornslaeger E, Mattei P \& Schultz R 1986 Involvement of cAMPdependent protein kinase and protein phosphorylation in regulation of mouse oocyte maturation. Developmental Biology 114 453-462.

Chesnel F \& Eppig J 1995 Induction of precocious germinal vesicle breakdown (GVB) by GVB-incompetent mouse oocytes. Possible role of mitogen-activated protein kinases rather than $\mathrm{p} 34^{\text {cdc2 }}$ kinase. Biology of Reproduction 52 895-902.

Cohen P 1978 The role of cyclic-AMP-dependent protein kinase in the regulation of glycogen metabolism in mammalian skeletal muscle. Current Topics in Cellular Regulation 14 117-196.

Cohen P 1989 The structure and regulation of protein phosphatases. Annual Review of Biochemistry 58 453-508.

Dohadwala M, da Cruz e Silva E, Hall F, Williams R, CarbonaroHall D, Nairn A, Greengard P \& Berndt N 1994 Phosphorylation and inactivation of protein phosphatase 1 by cyclin-dependent kinases. PNAS 91 6408-6412.

Endo S, Zhou X, Connor J, Wang B \& Shenoliker S 1996 Multiple structural elements define the specificity of recombinant human inhibitor-1 as a protein phosphatase-1 inhibitor. Biochemistry 35 5220-5228.

Erickson G \& Sorenson A 1974 In vitro maturation of mouse oocytes isolated from late, middle, and pre-antral Graafian follicles. Journal of Experimental Zoology 190 123-127.

Gavin A-C, Tsukitani Y \& Schorderet-Slatkine S 1991 Induction of M-phase entry of prophase-blocked mouse oocytes through microinjection of okadaic acid, a specific phosphatase inhibitor. Experimental Cell Research 192 75-81.

Goris J, Hermann J, Hendrix P, Ozon R \& Merlevede W 1989 Okadaic acid, a specific protein phosphatase inhibitor, induces maturation and MPF formation in Xenopus laevis oocytes. FEBS Letters 245 91-94.

Hampl A \& Eppig J 1995 Translational regulation of the gradual increase in histone $\mathrm{H} 1$ kinase activity in maturing mouse oocytes. Molecular Reproduction and Development 40 9-15.

Harrouk W \& Clarke H 1995 Mitogen-activated protein (MAP) kinase during the acquisition of meiotic competence by growing oocytes of the mouse. Molecular Reproduction and Development $\mathbf{4 1}$ 29-36.

Hashimoto H \& Kishimoto T 1988 Regulation of meiotic metaphase by a cytoplasmic maturation-promoting factor during mouse oocyte maturation. Developmental Biology 126 242-252.
Heikinheimo O, Lanzendorf S, Baka S \& Gibbons W 1995 Cell cycle genes C-mos and cyclin-B1 are expressed in a specific pattern in human oocytes and preimplantation embryos. Molecular Human Reproduction 10 699-707.

Hemmings B, Resink T \& Cohen P 1982 Reconstitution of a MgATPdependent protein phosphatase and its activation through a phosphorylation mechanism. FEBS Letters 150 319-324.

Ingebritsen T, Stewart A \& Cohen A 1983 The protein phosphatases involved in cellular regulation. 6. Measurement of type-1 and type-2 protein phosphatases in extracts of mammalian tissues; an assessment of their physiological roles. European Journal of Biochemistry 132 297-307.

Jagiello I, Beullens M, Stalmans W \& Bollen M 1995 Subunit structure and regulation of protein phosphatase- 1 in rat liver nuclei. Journal of Biological Chemistry 270 17257-17263.

Jessus C, Goris J, Staquet S, Cayla X, Ozon R \& Merlevede W 1989 Identification of the ATP + Mg-dependent and polycationstimulated protein phosphatases in the germinal vesicle of the Xenopus oocyte. Biochemical Journal $26045-51$.

Kawai J, Shinagawa A, Shibata $K$, Yoshino $M$, Itoh $M$, Ishii $Y$ Arakawa T, Hara A, Fukunishi Y, Konno H, Adachi J, Fukuda S, Aizawa K, Izawa M, Nishi K, Kiyosawa H, Kondo S, Yamanaka I \& Saito T 2001 Functional annotation of a full-length mouse cDNA collection. Nature 409 685-690.

Kwon Y-G, Lee S, Choi Y, Greengard P \& Nairn C 1997 Cell cycledependent phosphorylation of mammalian protein phosphatase 1 by cdc2 kinase. PNAS 94 2168-2173.

Laemmli U 1970 Cleavage of structural proteins during the assembly of the head of bacteriophage T4. Nature 227 680-685.

Leach C, Eto M \& Brautigan DL 2002 Domains of type 1 protein phosphatase inhibitor-2 required for nuclear and cytoplasmic localization in response to cell-cell contact. Journal of Cell Science 115 3739-3745.

Levesque J \& Sirard M 1995 Effects of different kinases and phosphatases on nuclear and cytoplasmic maturation of bovine oocytes. Molecular Reproduction and Development 42 $114-121$.

Mitra J \& Schultz R 1996 Regulation of the acquisition of meiotic competence in the mouse. Changes in the subcellular localization of cdc2, cyclin B1, cdc 25C and weel, and in the concentration of these proteins and their transcripts. Journal of Cell Science 109 $2407-2415$.

Naito K \& Toyoda Y 1991 Fluctuation of histone H1 kinase activity during meiotic maturation in porcine oocytes. Journal of Reproduction and Fertility 93 467-473.

Nimmo G \& Cohen P 1978 The regulation of glycogen metabolism. Purification and characterization of protein phosphatase inhibitor1 from rabbit skeletal muscle. European Journal of Biochemistry $\mathbf{8 7}$ 341-351.

Ohkura H, Kinoshita N, Miyatani S, Toda T \& Yanagida M 1989 The fission yeast dis2 + gene required for chromosome disjoining encodes one of two putative type 1 protein phosphatases. Cell $\mathbf{5 7}$ 997-1007.

Peter M, Nakagawa J, Doree M, Labbe J \& Nigg E 1990 In vitro disassembly of the nuclear lamina and $M$ phase-specific phosphorylation of lamins by cdc2 kinase. Cell 61 591-602.

Pincus G \& Enzmann E 1935 The comparative behavior of mammalian eggs in vivo and in vitro. I. The activation of ovarian eggs. Journal of Experimental Medicine 62 655-675.

Pondaven P \& Cohen P 1987 Identification of protein phosphatases-1 and $-2 \mathrm{~A}$ and inhibitor-2 oocytes of the starfish Asterias rubens and Marthasterias glacialis. European Journal of Biochemistry 167 135-140.

Resink T, Hemmings B, Tung H \& Cohen P 1983 Characterization of a reconstituted Mg-ATP-dependent protein phosphatase. European Journal of Biochemistry 133 455-461.

Rime H \& Ozon R 1990 Protein phosphatases are involved in the in vivo activation of histone $\mathrm{H} 1$ kinase in mouse oocyte. Developmental Biology 141 115-122. 
Schultz R \& Wassarman P 1977 Biochemical studies of mammalian oogenesis. Protein synthesis during oocyte growth and meiotic maturation in mouse. Journal of Cell Science 24 167-194.

Schwartz D \& Schultz R 1991 Stimulatory effect of okadaic acid, an inhibitor of protein phosphatases, on nuclear envelope breakdown and protein phosphorylation in mouse oocytes and one-cell embryos. Developmental Biology 145 119-127.

Smith G, Wolf D, Trautman K, da Cruz e Silva E, Greengard P \& Vijayaraghavan S 1996 Primate sperm contain protein phosphatase 1, a biochemical mediator of motility. Biology of Reproduction 54 $719-727$.

Smith G, Sadhu A, Mathies S \& Wolf D 1998a Characterization of protein phosphatases in mouse oocytes. Developmental Biology 204 537-549.

Smith G, Sadhu A \& Wolf D $1998 b$ Transient exposure of rhesus macaque oocytes to calyculin-A and okadaic acid stimulates germinal vesicle breakdown permitting subsequent development and fertilization. Biology of Reproduction 58 880-886.

Swain J, Wang X, Saunders T, Dunn R \& Smith G 2003 Inhibition of mouse oocyte nuclear protein phosphatase-1 stimulates germinal vesicle breakdown. Molecular Reproduction and Development 65 96-103.

Van Eynde A, Wera S, Beullens M, Torrekens S, Van Leuven F, Stalmans W \& Bollen M 1995 Molecular cloning of NIPP-1, a nuclear inhibitor of protein phosphatase-1, reveals homology with polypeptides involved in RNA processing. Journal of Biological Chemistry $27028068-28074$.

de Vantery C, Gavin A, Vassalli J \& Schorderet-Slatkine S 1996 An accumulation of $\mathrm{p} 34^{\mathrm{cdc} 2}$ at the end of mouse oocyte growth correlates with the acquisition of meiotic competence. Developmental Biology $174335-344$.

Vijayaraghavan S, Stephens D, Trautman K, Smith G, Khatra B, da Cruz e Silva E \& Greengard P 1996 Sperm motility development in the epididymus is associated with decreased glycogen synthase kinase-3 and protein phosphatase 1 activity. Biology of Reproduction 54 709-718.

Wang X, Liu XT, Dunn R, Ohl DA \& Smith GD 2003 Glycogen synthase kinase-3 regulates mouse oocyte homologue segregation. Molecular Reproduction and Development 64 96-105.

Zhao S, Xia W \& Lee E 1996 Affinity chromatography of regulatory subunits of protein phosphatase-1. Archives Biochemistry Biophysics $32582-90$.

Received 14 January 2004

First decision 1 April 2004

Revised manuscript received 23 June 2004

Accepted 5 July 2004 